\title{
Trabajo social en el contexto neoliberal global: solidaridad y resistencia desde una perspectiva radical
}

\section{Social work in the global neoliberal context: solidarity and resistance from a radical perspective}

\author{
Vasilios loakimidis ${ }^{1}$ \\ University of Essex, Inglaterra
}

Recibido: 03/09/2020

Aceptado: 10/11/2020

\section{Cómo citar}

loakimidis, V. (2021). Trabajo social en el contexto neoliberal global: solidaridad y resistencia desde una perspectiva radical. Propuestas Críticas en Trabajo Social - Critical Proposals in Social Work 1(1), 28-42. DOI: 10.5354/2735-6620.2021.61229

\section{Resumen}

El capitalismo neoliberal ha impactado de manera brutal en términos del Palabras clave: Neoliberalismo; incremento de la desigualdad en todo el mundo. Esto tiene una estrecha relación con los problemas de salud mental que enfrenta de manera creciente la población en general, incluyendo a las/os trabajadores sociales. En este artículo planteo que no puede haber un trabajo social crítico, basado genuinamente en trabajo social radical; relaciones humanas; la búsqueda de la justicia social, que no ponga énfasis en las relaciones humanas; resistencia. y que tampoco puede haber un trabajo social basado en las relaciones humanas que no aspire a la promoción de la justicia social en un plano estructural. Para argumentar en torno a esta posición, se abordan discusiones sobre el neoliberalismo y su impacto en las relaciones humanas como un marco amplio para pensar a trabajo social hoy. Se problematiza el pasado profesional y se analizan las condiciones actuales en que se produce la intervención de las/os 
trabajadores sociales, para pensar desde allí la posibilidad de la resistencia. En base al análisis de los actos de resistencia del trabajo social en algunos países europeos, se propone un proyecto radical de trabajo social, que pone al centro la solidaridad y el cuidado como impulso transformador en nuestras sociedades.perspectivas nos permiten considerar al lenguaje como principio ordenador y evidenciar la emergencia y actualidad del enfoque interpretativo. Planteamos esta lectura justamente porque consideramos que al trabajo social le ha costado, y le cuesta, incluirse en esta perspectiva, pese a las arraigadas tradiciones tanto técnico-instrumentales como teórico-epistémicas que abogan o promulgan la importancia de la interpretación. El final del texto abre a nuevas interrogantes, antes que a respuestas formalmente elaboradas. El punto de apertura y cierre, en espiral, es la reflexividad y la crítica.

\section{Abstract}

This article aims to work on the relationship between philosophy and epistemology with social work, assuming a genealogical reading of its evolution that will allow us to highlight the points of contact and the divergences. A critical analysis of the current positions on Mary Richmond's productions is presented, reflecting on her influences, her contributions and her positions, asking ourselves what is the connection between these interpretative views and the Keywords: critique; resistance; social work; neoliberalism. more positivist / functionalist epistemic positions of the social work, or even those oriented towards Marxism / dialectics, insofar as these two approaches have been, in different periods, hegemonic in the profession / discipline? To elaborate on this discussion, we refer to another disciplinary field: Ferdinand de Saussure's contributions to structural linguistics, alongside psychoanalysis. These perspectives allow us to consider language as an ordering principle and to show the emergence and relevance of the interpretive approach. We propose this reading precisely because we consider the difficulty social work has had to be included in this perspective, despite the ingrained technical-instrumental and theoretical-epistemic traditions that advocate or promote the importance of interpretation. The end of the text opens up new questions, rather than formally elaborated answers. The opening and closing point, in a spiral, is reflexivity and criticism. 


\section{Introducción}

En marzo de 2019 nuestra profesión celebró el Día Internacional del Trabajo Social bajo el lema "promover la importancia de las relaciones humanas". Este fue un tema muy bien recibido que generó, con razón, mucha discusión sobre la naturaleza de nuestra profesión y los vínculos que existen entre la forma en que los individuos y sus relaciones se configuran en diferentes contextos sociopolíticos. Esta observación nos lleva a la pregunta central que aquí se quiere discutir. En una profesión que históricamente ha concentrado la mayor parte de su actividad y energía en el trabajo con individuos y que en muchos aspectos ha descuidado lo que podríamos llamar el "nivel estructural", el "nivel macro" o el "trabajo social basado en la justicia social" ¿Por qué podría ser necesario dedicar el día mundial del trabajo social sobre este tema específico -las relaciones humanas- ¿No constituye esto una paradoja o, incluso, un anacronismo?

Mi respuesta a esas preguntas es decididamente negativa. La tesis que quiero defender en este artículo es que, por el contrario, no puede haber un trabajo social crítico, orientado por el principio de la justicia social, que no ponga énfasis en las relaciones humanas. Pero tampoco puede haber un trabajo social basado en las relaciones humanas que no aspire a la promoción de la justicia social a nivel estructural. Desde una perspectiva radical, estas dos dimensiones están íntimamente vinculadas y cualquier esfuerzo por separar lo uno de lo otro -las micropolíticas de la resistencia y el trabajo social crítico un plano estructural- reducirá inevitablemente el trabajo social a una actividad tecnocrática o a una abstracta actividad pseudopolítica. Estas comprensiones binarias de trabajo social son precisamente las que han alimentado los procesos de desprofesionalización (Ferguson et al., 2018) que hemos venido experimentando desde las últimas décadas en diversos países del mundo.

En este artículo abordaré tres áreas principales e interrelacionadas que se derivan de esta tesis, de manera de reflexionar sobre lo que implica pensar la solidaridad y las resistencias desde trabajo social en el contexto neoliberal global. En primer lugar presentaré un análisis respecto a cómo el neoliberalismo afecta las relaciones humanas y la salud mental de las personas. En segundo lugar analizaré elementos de la economía política del trabajo social, discutiendo los impactos del neoliberalismo en las condiciones laborales de las/os trabajadores sociales en base a los resultados de un estudio realizado en Reino Unido por la Asociación Británica de Trabajadoras/es Sociales en 2019. En tercer lugar, y con el propósito de avanzar hacia un replanteamiento del trabajo social desde una perspectiva radical, revisaré críticamente algunos pasajes de la historia profesional que nos permitan problematizar y replantear los principios de justicia social de trabajo social. Finalmente, presentaré algunas propuestas que se enmarcan en lo que en Reino Unido y otros países europeos se 
denomina enfoque radical de trabajo social (Ferguson et al., 2018), incluyendo una reflexión sobre la relevancia de las alianzas internacionales y el compromiso con las demandas de colectivos y movimientos sociales como parte de la agenda política de la profesión y disciplina.

\section{Las relaciones humanas en el neoliberalismo}

En los últimos años, nuestras sociedades, que han sido reformadas agresivamente como economías orientadas al mercado, experimentaron una nueva epidemia sin precedentes y abrumadora: el sufrimiento mental.

Según la Organización Mundial de la Salud, OMS (2017), solo en los países de la Unión Europea (UE), Islandia, Noruega y Suiza, el 27\% de la población adulta (aquí definida como de 18 a 65 años de edad) había experimentado al menos uno de una serie de problemas de salud mental en el año anterior a la consulta (esto incluía problemas derivados del uso de sustancias, psicosis, depresión, ansiedad y trastornos de la alimentación). Las tasas de angustia para las mujeres fueron significativamente más altas en comparación con las de los hombres (33\%). Los datos también mostraron que estos problemas de salud mental afectaron de manera mucho más significativa a las personas de hogares de más bajos ingresos, a los desempleados y a las personas que reciben beneficios estatales.

Los problemas de salud mental no son nuevos, por supuesto. Se han observado y experimentado desde la creación de las primeras comunidades humanas. Sin embargo, la pregunta importante, desde la perspectiva de trabajo social, es qué explica realmente el enorme aumento de la angustia que se experimenta en el mundo occidental hoy en día. Me refiero a los factores que inciden en que la angustia y otros problemas de salud mental se intensifiquen en ciertos segmentos de la población, los sectores más empobrecidos.

Los puntos de vista tradicionales sobre la angustia y los problemas de salud mental, que también han influido enormemente en el trabajo social, no han podido explicar completamente este aumento (Hart et al., 2019). Esto se debe a que la atención se ha centrado en la patología individual, tratando de explicar los problemas de salud mental de manera similar a la enfermedad física, a menudo atribuyendo los síntomas a desequilibrios químicos $\mathrm{u}$ hormonales o, más recientemente, priorizando una comprensión neurológica del desarrollo de los individuos. Es lo que tradicionalmente hemos conocido como enfoque biomédico, una de las bases teóricas dominantes de la formación disciplinar en trabajo social. Ciertamente, el enfoque biomédico no siempre captura la causa basal de la angustia. No permite un análisis más significativo en relación al contexto epidemiológico, social y político más amplio, que lleva a un 
aumento de la angustia en ciertas sociedades más que en otras, o en ciertos períodos históricos más que en otros (como el actual).

Como Ian Ferguson ha mencionado en su reciente libro "Las políticas de la mente" (2017), el modelo biomédico individualiza la angustia -o, en otras palabras, centra la comprensión del fenómeno de la angustia en la/el individuo que la vive. El punto de partida, desde una perspectiva radical en trabajo social, es desafiar esa creencia que sigue arraigada y que se reproduce cotidianamente en las intervenciones profesionales. Desafiar esa matriz biomédica, neutral y aséptica, implica comprender que el aumento significativo de los niveles de angustia está estrechamente relacionado con la presión que el capitalismo neoliberal ejerce sobre la vida de las personas.

A esto agregaría como factor adicional fundamental la desigualdad social. Los investigadores Wilkinson y Pickett (2010), en su extenso trabajo epidemiológico sobre la desigualdad, han confirmado lo que generaciones de trabajadoras/es sociales han presenciado en la primera línea de su intervención profesional: son las circunstancias materiales las que principalmente moldean las vidas de las personas, no su moralidad. Su libro destaca los horribles efectos que la desigualdad tiene en las sociedades: erosiona la confianza, aumenta la ansiedad y la enfermedad y fomenta el consumo compulsivo y excesivo. Con referencia a la salud mental, los investigadores han sugerido que hasta hace poco era difícil comparar los niveles de los problemas de salud mental entre diferentes países porque nadie había recopilado datos estrictamente comparables, pero recientemente la Organización Mundial de la Salud ha establecido encuestas mundiales de salud mental que están comenzando a proporcionar datos. Estos muestran que las diferentes sociedades tienen niveles muy diferentes de problemas de salud mental. En algunos países, cerca del 5\% de la población adulta ha sufrido algún problema de salud mental en el último año, pero en los Estados Unidos, más del $25 \%$ lo ha hecho.

En su investigación, Wilkinson y Pickett mostraron una relación entre los problemas de salud mental y desigualdad de ingresos en ocho países desarrollados, con datos de la OMS: Estados Unidos, Francia, Países Bajos, Bélgica, España, Alemania, Italia y Japón. La conclusión apunta a que los problemas de salud mental son mucho más frecuentes en países más desiguales. También se observó que los problemas de salud mental eran más comunes en los países más ricos incluidos en el estudio.

Se ha observado un patrón similar en diferentes variables como el crimen, la obesidad, la salud física, entre otras. Hoja por hoja y caso tras caso esta investigación demuestra que las sociedades más desiguales crean individuos más enfermos e infelices. Por lo tanto, mejorar las relaciones humanas es un asunto colectivo y no individual, que 
requiere cambios estructurales en la forma en que se organiza la economía de nuestras sociedades y no cambios de conductas meramente individuales.

El impacto catastrófico de la desigualdad se ha exacerbado en gran parte del mundo debido a los efectos de la "recesión prolongada" que tuvo lugar en 2008. Para ser más preciso, la desigualdad social se ha exacerbado específicamente debido a las decisiones ideológicas impulsadas por los gobiernos y el Fondo Monetario Internacional (Ioakimidis et al., 2014). La trinidad santísima del neoliberalismo (mercantilización, privatización y austeridad) fue nuevamente invocada como respuesta a la crisis global. Los efectos de esa "larga recesión" se han experimentado, por supuesto, de manera muy diferente en diferentes sectores de la población mundial.

Las políticas de austeridad - la disminución al máximo del gasto social en los desmantelados estados de bienestar europeos- ha sido la estrategia económica, ideológica y política de corto plazo que ha dominado Europa durante la mayor parte de la última década. Su atractivo para los gobiernos es que parece proporcionar una explicación clara, simple y moralista para la crisis actual. Por ejemplo, se ha aducido la existencia de un gasto gubernamental excesivo, especialmente en asistencia social donde miles de personas flojas o work-shy (tímidas frente trabajo) estarían supuestamente aprovechándose del Estado.

La solución a esa crisis, como sugiere este análisis simplista, es reducir los salarios, reducir el gasto público y aumentar los impuestos. En casi todos los casos, esa solución también ha implicado una "reforma estructural", lo que significa una mayor flexibilidad del mercado, reducciones de las pensiones, la privatización de empresas públicas, etcétera. Un informe de Oxford Committee for Famine Relief (Oxfam), publicado a principios de 2016 mostró que solo el 1\% de la población mundial posee actualmente más riqueza que todo el resto del mundo junto. Aún más crudamente, 62 personas poseen tanta riqueza como la mitad más pobre de la población mundial. "Una economía para el 1\%" mostró que la riqueza de la mitad más pobre de la población mundial, 3.600 millones de personas, ha disminuido en un billón de dólares desde 2010. Esta caída del $38 \%$ se produjo a pesar de que la población mundial aumentó en alrededor de 400 millones de personas durante ese período. Mientras tanto, la riqueza de los 62 más ricos se ha incrementado en más de medio trillón de dólares a 1.76 billones de dólares (Oxfam, 2016).

\section{Las condiciones laborales de los trabajadores sociales}

En este escenario de desmantelamiento del bienestar en Europa, la alienación, la intensificación del trabajo y la atomización que caracterizan la mercantilización agresiva se reflejan en el deterioro de la salud mental de las personas. En relación con 
las condiciones de trabajo de las/os trabajadores sociales, esto se vuelve aún más evidente a medida que la liberalización de nuestras economías ha creado empleos inseguros, intensivos y mal pagados.

Esto nos lleva a la segunda razón principal por la que hacer hincapié en las relaciones humanas es una decisión oportuna y significativa, más aún si nos situamos desde un enfoque radical en trabajo social. Si asumimos que fomentar una relación con las personas con las que trabajamos es un proceso que implica la participación activa y proactiva de ambos lados, y que cualquier aspecto de la vida personal o profesional que afecte a ambos lados debe considerarse, no podemos ignorar las condiciones específicas que los trabajadores sociales experimentan en sus empleos. Ya describimos el panorama general e identificamos la salud mental y las presiones económicas que viven muchas de las personas con las que trabajamos. Pero ¿qué pasa con las condiciones específicas que experimentan las/os propios trabajadores sociales (y su impacto inevitable en su calidad de vida, así como en sus relaciones con los usuarios de los servicios)?

Obviamente, estas condiciones tienden a variar de un país a otro, pero el asunto aquí es que las/os trabajadores sociales no eligen su profesión porque quieren enriquecerse -si hubiesen querido hacerse ricos, entonces el trabajo social presumiblemente no hubiese sido la carrera correcta para ellos-. La mayoría de las/os trabajadores sociales eligen su profesión principalmente porque están comprometidos con la justicia social y quieren lograr una transformación en la vida de las personas. Sin embargo, la forma y la función de las economías neoliberales afectan las experiencias de trabajo social en sus empleos. $\mathrm{Si}$ bien somos una profesión de rápido crecimiento en términos de números e influencia, todavía hay mucho que se necesita lograr con respecto a las condiciones de trabajo.

En un estudio reciente encargado por la Asociación Británica de Trabajadores Sociales en 2018 (Ravalier \& Boichat, 2018), fue la angustia reportada por los propios trabajadores sociales lo que más llamó la atención. Y los resultados fueron crudos y alarmantes:

- En comparación con el promedio del Reino Unido, las condiciones de trabajo de las/os trabajadores sociales eran peores que las del $95 \%$ de otros empleados que se desempeñan tanto en el sector público como en el privado,

- Casi la mitad de las/os trabajadores sociales declaran no estar satisfechos con sus trabajos, 
- Dos tercios de ellas/os han asistido a trabajar mientras estaban enfermos. Lo han hecho al menos dos veces en el último año,

- Las/os trabajadores sociales trabajaron un promedio de 64 días por año por sobre lo que estaban contratados (un promedio de 11 horas extra no remuneradas por semana),

- El 60\% de las/os trabajadores sociales declaró que deseaba dejar su trabajo actual en los próximos 15 meses, en comparación con el 52\% reportado el año pasado.

- Casi el 40\% de las/os encuestados buscan abandonar la profesión por completo.

- Los principales factores estresantes identificados por las/os participantes fueron las altas cargas administrativas y de casos que intervenir, además de la angustia al ver la falta de recursos para brindar mejores atenciones a las/os usuarios.

Lo interesante aquí es que, en muchos aspectos, las/os trabajadores sociales enfrentan condiciones no muy diferentes a las situaciones que experimentan los usuarios de nuestros servicios (alienación, angustia, trabajos inseguros, etcétera).

Esta observación me lleva al argumento principal que quiero plantear: si queremos lograr un cambio a través del fomento de las relaciones humanas transformadoras, debemos repensar el trabajo social y desarrollar modelos críticos, integrales, no estigmatizantes y anti-opresivos.

\section{Un pasado complejo}

La tercera razón que resalta la importancia de reclamar y reimaginar el trabajo social radical en la era del capitalismo neoliberal está relacionada con nuestra propia historia como profesión (Ioakimidis y Trimikliniotis, 2020).

Debemos recordar y celebrar a las/os trabajadores sociales que fueron pioneros en la promoción de los derechos humanos. Desde el movimiento de asentamientos del siglo XIX en América del Norte hasta el movimiento de reconceptualización en América Latina, desde la resistencia de las comunidades indígenas hasta la creación de la Red de Acción del Trabajo Social -SWAN por sus siglas en inglés-, ha habido una historia fascinante de crítica en trabajo social que, aunque en gran medida inexplorada en el mundo anglo-americano, ha influenciado sustantivamente a la profesión. Muchas pioneras del trabajo social promovieron los derechos humanos y corrieron un gran riesgo para sus propias vidas al ser perseguidas, encarceladas y asesinadas. Un 
inspirador ejemplo de ello son aquellas trabajadoras sociales chilenas cuyo compromiso con la democracia y la justicia salvó vidas durante la brutal dictadura de Pinochet (Muñoz Arce, 2020).

Sin embargo, también debemos explorar aquellas partes de nuestra historia en que las/os trabajadores sociales han sido cómplices en algunos de los hechos más horribles que la humanidad ha presenciado en el siglo veinte. Varios incidentes históricos destacan ejemplos de notable brutalidad, informados por el desarrollo de coyunturas políticas igualmente extraordinarias. En Europa, estos casos pueden vincularse, sobre todo, con el surgimiento de ideologías fascistas y nazis y su preocupación pseudocientífica por la creación de una "raza superior" a través de la eugenesia. Algunas/os trabajadores sociales y pedagogos sociales estuvieron directamente involucrados en el proceso de monitoreo de la organización de las familias y el adoctrinamiento de las/os niños.

Desafortunadamente, la instrumentalización de la eugenesia en el contexto de los servicios sociales no terminó con la Segunda Guerra Mundial. Hasta aproximadamente la década de 1970, los problemas sociales en los Estados Unidos como la pobreza, el crimen y el desempleo, se consideraron en gran parte "hereditarios" dentro de las clases sociales empobrecidas y, por lo tanto, se trataron mediante prácticas dirigidas a evitar que estas clases se "reprodujeran". Investigaciones recientes sugieren que en algunos estados (especialmente en Carolina del Norte) esta práctica duró hasta bien entrada la década del 1970 y afectó a más de 7,600 familias que vivían en la pobreza y pertenecían a grupos étnicos minoritarios (Ioakimidis y Trimikliniotis, 2020).

El trabajo social colonial también proporcionó un terreno fértil para las violaciones de los derechos humanos. Por ejemplo, en Australia, Canadá y Nueva Zelanda, los pueblos originarios, y los padres, madres y familias de pueblos originarios se consideraron "no aptos" para educar a sus hijos. Como resultado, solo entre 1971 y 1981, más de 3,400 niños indígenas fueron enviados a padres adoptivos en otras sociedades, y algunas veces en otros países.

Necesitamos ser valientes y tener confianza al explorar nuestra propia historia y, en particular, los capítulos específicos de esta historia que demostraron que el trabajo social basado en las relaciones puede caer fácilmente en una grave violación de los derechos humanos si no toma en cuenta de manera más amplia el contexto estructural ideológico al que sirve. 


\section{Trabajo social basado en las relaciones humanas: una perspectiva radical}

Parte de la discusión actual sobre el trabajo social, su sentido y sus posibilidades en medio del capitalismo neoliberal, se refleja en una pregunta crucial: ¿cómo definimos lo que hacemos? De hecho, las diferentes formas en que las organizaciones internacionales del trabajo social y los colectivos y movimientos sociales que se han involucrado en este debate revelan las tensiones ideológicas que dividen el proyecto de trabajo social. Esto no es simplemente un debate teórico o abstracto: definir el trabajo social tiene un impacto en lo que sucede en la intervención profesional. Cuando enfatizan las interpretaciones individualistas y moralistas del trabajo social, estas tienden a reducir el trabajo social a una actividad técnica meramente formal.

Es por esta razón que tenemos que repensar radicalmente el trabajo social. De una manera que abarque las relaciones humanas, pero que al mismo tiempo aprecie la importancia de la estructura social y política más amplia. Las/os trabajadores sociales deben comprender y abordar no solo los síntomas de la angustia, sino principalmente las causas públicas del dolor y la desdicha.

A pesar de las recientes desviaciones y malas interpretaciones del término, el concepto "radical" históricamente se ha referido a una teoría y práctica política que tiene como objetivo comprender y apuntar a las causas estructurales de los problemas sociales (Ferguson et al., 2018). En el contexto del bienestar social, no es raro que las políticas estatales promuevan valores exactamente opuestos a esta perspectiva e ignoren las causas estructurales de las dificultades que experimentan las/os usuarios de los servicios sociales. Por ejemplo, todavía es posible escuchar que los pobres son pobres porque son flojos, que las mujeres se embarazan porque quieren recibir beneficios del estado o que los refugiados están excluidos porque no quieren aceptar nuestra cultura, e incluso que se ridiculice a las personas con problemas de salud mental. Lo que quiero marcar es que, si ignoramos la desigualdad y la pobreza como factor estructural, nuestra intervención profesional se reduce a una función inútil y superficial, como si se tratase de una "aspirina" social.

En el trabajo social radical, basado en la justicia social, el uso de diversos métodos y técnicas (como el trabajo con colectivos, las intervenciones basadas en el arte, la promoción, la sensibilización, el trabajo con casos desde perspectivas críticas y la acción social con las comunidades, entre muchísimas otras) está orientado a apoyar a las víctimas de un sistema desigual, pero también a crear las condiciones para la emancipación y resistencia frente al orden aparentemente natural de nuestras sociedades. Eso conduciría a la creación de sociedades socialmente justas. 
Como mencioné anteriormente, la economía neoliberal y las prácticas opresivas no han sido suficientemente impugnadas. Pero a pesar de la política del miedo que se ha expandido por los distintos rincones de ese mundo, en muchos países se ha visto una resistencia extraordinaria por parte de las/os trabajadores sociales. Esto da forma a lo que podemos llamar una "política de la esperanza", en la que la solidaridad constituye una forma de resistencia dentro de un sistema que pugna por la competencia y el individualismo en todos los dominios de la vida.

En muchas ocasiones, los trabajadores sociales han liderado estas iniciativas ofreciendo ejemplos maravillosos de cómo debería ser un estado inclusivo, participativo y de bienestar democrático. Por ejemplo, en España, durante la crisis financiera y las reformas neoliberales draconianas que siguieron, los trabajadores sociales fueron muy activos en el movimiento La Marea Naranja (Ioakimidis et al., 2014). Este movimiento hizo hincapié en la resistencia frente a los recortes y en la demanda de más recursos para los servicios sociales y reunió a grupos e instituciones muy diversas relacionadas con el bienestar.

En Grecia, durante el mismo período, las/los trabajadores sociales cometieron actos de “desobediencia civil" (Ioakimidis y Teloni, 2013). Cuando el gobierno impuso un impuesto principal regresivo y horizontal pagadero a través de las cuentas de electricidad, las/los trabajadores sociales desobedecieron la instrucción de trabajar con los recaudadores de impuestos para identificar a los hogares que tendrían dificultades para pagar. Al desobedecer la ley, se aseguró que protegían la dignidad y los derechos de los más pobres de la sociedad y no estaban involucrados en esta política deshonesta, impopular y opresiva. Por el contrario: deslegitimaron la política y se unieron al movimiento social que se oponía a los recortes de fondos.

Por último, pero no menos importante, los trabajadores sociales en Gran Bretaña sabían muy bien que las medidas de austeridad y la privatización de los servicios sociales que implementaba el gobierno conservador de Cameron, en 2010, conducirían a una catástrofe social y, por eso, presionaron ampliamente a los miembros del parlamento, pero también se movilizaron a nivel de base. Un movimiento admirable e inspirador, el movimiento contra la austeridad, apoyado por la Asociación Británica de Trabajadores Sociales, reunió a un grupo de colegas de trabajo social, usuarias/os y académicas/os -todas personas que han experimentado de primera mano el impacto de los recortes en el gasto público y la reforma de la asistencia social-, para marchar 100 millas desde Birmingham a Liverpool, protestando contra las políticas de austeridad bajo el lema Boot Out Austerity. Se trató de un excelente ejemplo de organización política del trabajo social de abajo hacia arriba, dirigido a la defensa de los servicios sociales. 
La oposición al fundamentalismo del mercado en la política social está arraigada en dos factores: primero, la certeza en que la política social y el estado de bienestar deben preocuparse principalmente por atender las necesidades humanas en lugar de impulsar la competencia, eficiencia y beneficio del mercado; y, segundo, la conciencia de que las formas neoliberales de trabajo social, incluida su dominación por procesos de evaluación y registro sin sentido, que se almacenan en una computadora sin reparar en lo sustantivo, han socavado gravemente las posibilidades de desarrollar una acción profesional crítica.

Como ya he mencionado, una de las víctimas de esta racionalidad neoliberal han sido las relaciones humanas en la provisión de los servicios sociales. Otra víctima ha sido el trabajo con colectivos y con comunidades. Alguna vez fue una parte clave de las respuestas políticas a la pobreza en Gran Bretaña y en otros países. Los enfoques de trabajo social basados en la comunidad, particularmente aquellos que promueven que la comunidad se "auto-ayude", pueden, por supuesto, ser al menos tan conservadores como los enfoques basados en el individuo. Sin embargo, un enfoque radical del trabajo con las comunidades, así como con los movimientos sociales, ofrece claras posibilidades para abordar las desigualdades estructurales y para resaltar el vínculo entre los problemas privados y los problemas públicos.

Como parte de este proceso, las/os trabajadores sociales deben utilizar la evidencia que viene de su propia intervención e investigación, para enfatizar y reclamar la necesidad de un estado inclusivo, redistributivo y universal (Ioakimidis, 2013).

Este formidable cuerpo de evidencia que los trabajadores sociales pueden levantar desde sus intervenciones e investigaciones conforma una base de conocimiento que reafirma el valor del universalismo y la solidaridad en la política social. Esto significa reclamar la necesidad de garantizar la amplia gama de derechos sociales y de servicios que permitan cubrir a toda la población en las diferentes etapas de la vida, donde existan criterios para priorizar por la infancia, las personas con alguna discapacidad, las personas mayores, etcétera, pero siempre desde una lógica universal de provisión de servicios sociales, inspirada por los principios de la redistribución, el reconocimiento y la defensa incondicional de la democracia.

\section{Conclusiones}

Contra aquellos que niegan que las/os trabajadores sociales desempeñan un papel en la lucha contra la opresión y por una sociedad más igualitaria, sostengo, por el contrario, que sí tenemos la capacidad y potencialidad para hacerlo. Sin embargo, dada la brutalidad de las fuerzas en contra de todos quienes buscamos construir un mundo mejor o simplemente defender el carácter universal de los derechos, no debemos 
hacernos ilusiones con respecto a la contribución que las profesiones, a veces débiles y desorganizadas, puedan hacer.

Por eso es sumamente importante que los profesionales formen alianzas con organizaciones sociales, sindicatos, con los colegios profesionales de trabajo social, con organizaciones de usuarios de servicios, para promover alternativas.

Como ejemplo, en el Reino Unido, la Red de Acción de Trabajo Social -SWAN(Ferguson et al, 2018) se ha vinculado con la Asociación Británica de Trabajadores Sociales, la Asociación de Personas con Discapacidad Contra los Recortes al gasto social, Disabled People Against Cuts, y la organización de usuarias/os de los servicios sociales Shaping Our Lives, para denunciar públicamente los ajustes de las políticas de austeridad, y para hacer campañas más efectivas contra sus efectos. Reforzar estas redes y aprender de la experiencia de profesionales, académicas/os, estudiantes, usuarias/os de servicios y activistas de campañas en diferentes países es una prioridad. Sin embargo, esto no se trata solo de compartir información, también se trata de mostrar solidaridad. Todos nos beneficiamos al desarrollar la solidaridad.

Debemos reclamar la solidaridad como un valor central. Reafirmar nuestra humanidad común no solo es la manera más efectiva de desafiar a los fundamentalistas, racistas y xenófobos del mercado, también desafía el individualismo estrecho y egoísta que reproducimos en nuestros comportamientos día a día.

Finalmente, y para cerrar y abrir al mismo tiempo estas reflexiones, quisiera recordar que nuestra definición global de trabajo social plantea que "el trabajo social promueve el cambio social y el empoderamiento y la liberación de las personas" (International Federation of Social Workers, IFSW, 2014). Tengan confianza en la capacidad que las personas y las sociedades tienen para cambiar. Trabajo social, cuando es democrático y empático, desde la crítica radical que no pierde la humanidad ni el cuidado por las relaciones humanas, puede tener un impacto transformador en individuos y sociedades. Y esto solo es una gran razón para sentirse orgulloso de nuestra profesión a lo largo y ancho de todo nuestro mundo.

\section{Referencias bibliográficas}

Ferguson, I. (2017). Politics of The Mind; Marxism and Mental Distress. Bookmarks.

Ferguson, I., Ioakimidis, V. \& Lavalette, M., (2018). Global Social Work in a Political Context, Radical Perspectives. Policy Press. 
Hart, E., Greener, J. \& Moth, R. (2019). Resist the Punitive State: Grassroots Struggles Across Welfare, Housing, Education and Prisons. Pluto Press.

International Federation of Social Workers. (2014). Global Definition of the Social Work Profession. https://www.ifsw.org/what-is-social-work/global-definition-of-social-work/

Ioakimidis V. \& Trimikliniotis, N. (2020). Making Sense of Social Work's Troubled Past: Professional Identity, Collective Memory and the Quest for Historical Justice. The British Journal of Social Work. https://doi.org/10.1093/bjsw/bcaa040

Ioakimidis,V., Cruz Santos, C. \& Martinez Herrero, I. (2014). Reconceptualizing Social Work in times of crisis: An examination of the cases of Greece, Spain and Portugal. International Social Work, 57(4), 285-300.

Ioakimidis, V. (2013). Beyond the dichotomies of cultural and political relativism: arguing the case for a social justice based 'global social work' definition. Critical and Radical Social Work, 1(2), 183-199.

Ioakimidis, V. \& Teloni, D. (2013). Greek social work and the never-ending crisis of the welfare state. Critical and Radical Social Work, 1(1), 31-49.

Muñoz Arce, G. (2020). Chilean Social Work and the Legacies of the Dictatorship. Social Dialogue, 22. https://www.iassw-aiets.org/es/get/social-dialogue-magazine-issue-22/

Organización Mundial de la Salud. (2017). Monitoring mental health systems and services in the WHO European Region: Mental Health Atlas, 2017.

https://www.euro.who.int/_data/assets/pdf_file/0009/396765/EMH-Atlas-6.pdf

Oxford Committee for Famine Relief. (2020). Time to care; Unpaid and underpaid care work and the global inequality crisis.

https://oxfamilibrary.openrepository.com/bitstream/handle/10546/620928/bp-time-to-care-ine quality-200120-en.pdf

Ravalier, J. \& Boichat, C. (2018). UK Social Workers: Working Conditions and Wellbeing. https://www.basw.co.uk/system/files/resources/Working\%20Conditions\%20\%20Stress\%20\% $282018 \% 29 \% 20$ pdf.pdf

Wilkinson, R.G. \& Pickett, K. (2010). The Spirit Level: Why Equality is Better for Every-one. Penguin. 


\section{Biografía del autor}

Vasilios Ioakimidis es Profesor Titular del Centre for Social Work, University of Essex, Reino Unido y Coordinador de la Comisión de Educación Global de la Federación Internacional de Trabajadoras/es Sociales. Correo electrónico:

v.ioakimidis@essex.ac.uk. Registro ORCID: https://orcid.org/0000-0002-0457-6782 